\title{
ESTENOSE ESQUISTOSSOMÓTICA DO INTESTINO DELGADO (RELATO DE UM CASO) *
}

\author{
Donald Huggins * *
}

\begin{abstract}
o Autor relato o caso de um paciente portador de estenose do intestino delgado (ileo iermival) cuịo aspecto macroscópico da peça operatória sugeriu o dixgnóstico de carcinoma, porém o exame histopatológico mostrou apenas lesao inflamatoria caüsada pelo Schistosoma mansoni.
\end{abstract}

\section{INTRODUÇÃO}

A esquistossomose mansônica é uma parasitose de elevada incidência em nosso meio, ocasionando em determinados indivíduos, o sindrome de hipertensão-porta, principalmente em adultos jovens, incapacitando-os para o seu trabalho habitual e propiciando uma substancial diminuição da mão-de-obra na área canavieira do Es.. tado (Pernambuco, Brasil).

Em raras ocasiōes, a bilharziose pode assumir um quadro clínico completamente diferente do habitualmente observado, traduzindo-se por sintomas cutâneos, neurológicos, genito-urinários e de oclusão intestinal.

$\mathrm{Na}$ oportunidade é relatado um caso de estenosa do intestino delgado que, apesar de procedente de uma zona altamente cndêmica da parasitose (Catende, Pernambuco, Brasil), o diagnóstico da mesma não foi lembrado.

Assim, pretendemos com a descrição do nosso doente, chamar a atenção para as formas clínicas anômalas da esquistosso- mose mansônica e, portanto, cogitá-la no diagnóstico diferencial dessas várias patologias, com o intuito de serem realizadas precocemente reações imunológicas para o seu diagnóstico e conseqüentemente efetuarmos o tratamento específico da parasitose a fim de evitar-se em alguns enfermos cirurgias mutilantes e inúteis.

\section{RELATO DO CASO}

S.T.L., 43 anos, masculino, melanodermo, natural de Catende (Pernambuco), trabalhador rural, foi admitido no Hospital das Clínicas da F.M.F.Pe. (Disciplina de Doenças Infecciosas e Parasitárias), queixando-se há três semanas de dor abdominal, localizada ao nível do hipogastro, de grande intensidade, propagando-se para o hipocôndrio e flanco esquerdos, acompanhada de ondas peristáticas visíveis com orientação da direita para a esquercia e constipação intestinal por 3 a 4 dias. Concomitantemente, referiu astenia, meteorismo, distensão abdominal e emagrecimento (aproximadamente $18 \mathrm{Kg}$ ) em virtude de

\footnotetext{
* Disciplina de Doenças Infecciosas e Parasitárias da Faculdade de Meöicina da Universidade Federa de Pernambuco, Brasil.

* Prof. Adjunto.

Recebido para publicação em 13-8-1972.
} 




Figura 1 - Paciente S.T.L. - Eletroforose das proteinas sericas. Notar a hipcalbuminemia e a hipergamaglobulinemia.

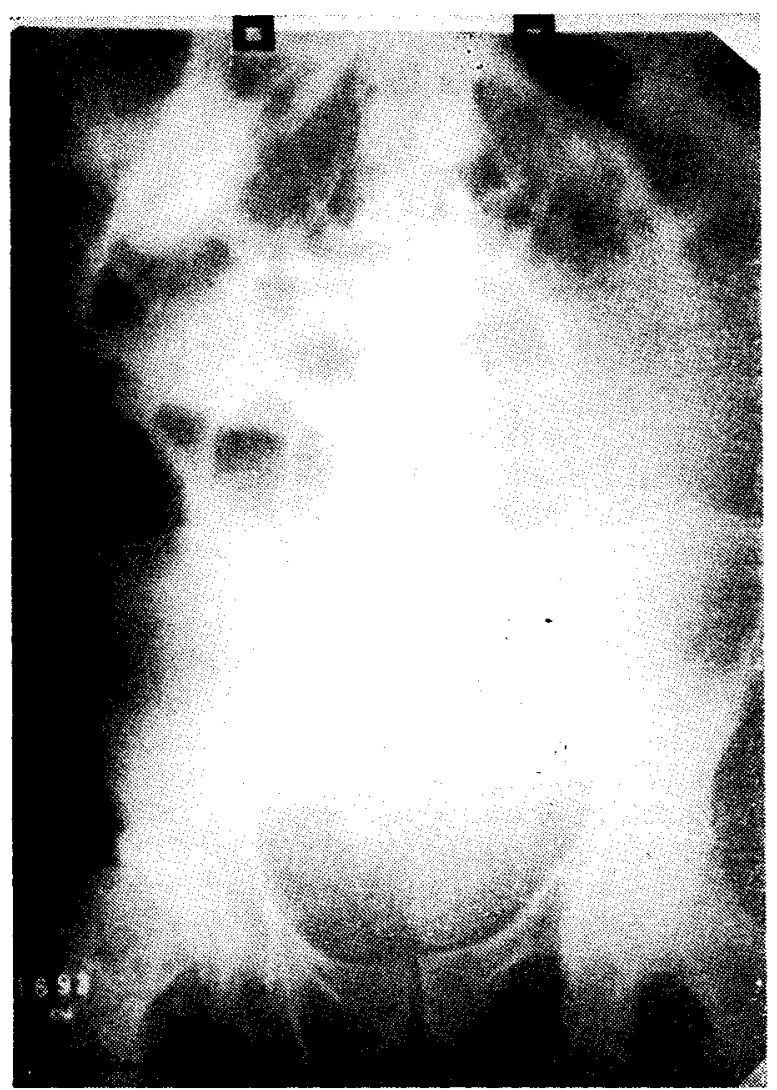

Figura 2 - Paciente S.T.L. - Estucio radiológico simples do abdome: verificar dilataçāo das alças jejunais e niveis hidro-aereos. 
fazer restrição alimentar por conta própria, devido a piora dos sintomas quando se alimenta.

Alimentação deficiente em proteinas; etilista eventual; banhos em rios desde a infância (rio Cupira - Catende, Pernambuco) e diz que já foi sugado pelo transmissor da doença de Chagas.

Ao exame físico apresenta estado geral regular, emagrecido (peso atual de $52 \mathrm{Kg}$; anterior de $70 \mathrm{Kg}$ ) ; apirético; facies atipica; marcha e fala normais; decúbito indiferente; atitude ativa; fôrça muscular conservada; mucosas coradas e T.A.: 120 a $80 \mathrm{mmm} \mathrm{Hg}$.

Aparelhos respiratório e cardiovascular - Clinicamente normais. Ap. digestivo: abdome distendido $(++)$, simétrico, tensão aumentada, dor $(++)$ ao nível do flanco direito, à palpação profunda, com desencadeamento de ondas peristálticas visíveis, localizadas nas regiōes umbilical e hipogastro. Fígado e baço não palpáveis; Traube - timpânico.

Demais aparelhos $e$ sistemas clinicamente normais.

Exames Complementares - Glicemia: $90 \mathrm{mg} / 100 \mathrm{ml}$; uréia: $25 \mathrm{mg} / 100 \mathrm{ml}$; creatinina: $1,40 \mathrm{mg} / 100 \mathrm{ml}$; sumário de urina: densidade $1009, \mathrm{pH} 5$, piocitos 19/campo e hematias 3/campo; eritrocitos: 4.290.00; hematocrito: $42 \%$; hemoglobina: $13 \mathrm{~g} / 100 \mathrm{ml}$; leucocitos: 8.000 (eosinófilos) : $21 \%$ ) ; plaquetas: 250.000 ; reticulocitos $1 \%$ $\left(42,900 / \mathrm{mm}^{3}\right)$; retração do coagulo: $58 \%$ após 2 horas; TC: $5,45 \mathrm{seg}$; TS: 2'; protrombina: $71 \%$ (15 segundos); parasitológico das fezes: ovos viáveis de Schistosoma mansoni e Ancylostomidae $s p$; coprocultura: Escherichia coli e Proteus rettgeri; protidograma (figura 1) - Proteina total: $6,48 \mathrm{~g} / 100 \mathrm{ml}$; albumina: $2,28 \mathrm{~g} / 100$ $\mathrm{ml}(35,26 \%)$; alfa 1 globulina: $0,25 \mathrm{~g} / 100$ $\mathrm{ml}(3,86 \%)$; alfa 2 globulina: $0,44 \mathrm{~g} / 100 \mathrm{ml}$ $(6.76 \%)$; beta globulina: $0,46 \mathrm{~g} / 100 \mathrm{ml} \mathrm{e}$ gama-globulina: $3,03 \mathrm{~g} / 100 \mathrm{ml}(46,37 \%)$ : sódio: $137 \mathrm{mEq} / 1$; potassio: $4,7 \mathrm{mEq} / 1$; cloro: $105 \mathrm{mEq} / 1$; reserva alcalina: 22,29 $\mathrm{mEq} / 1$; estudo radiológico do torax: normal; estudo radiológico simples do abdome (figura 2): alças cilatadas e níveis hidro-aéreos (sugestivo de processo oclusivo intestinal alto.)

Com o diagnóstico clínico de suboclusão intestinal alta, encaminhamos o paciente à 4a Clínica Cirúrgica Abdominal (Serviço do Prof. Salomão Kelner), onde foi submetido a ressecção enterica $(49 \mathrm{~cm}$ de ileo terminal) e anastomose termino-terminal pelo Dr. Pôrto Carreiro Junior.

A peça cirúrgica foi enviada para o serviço de Anatomia e Fisiologia Patológicas (Prof. R. Barros Coelho) e o estudo histopatológico realizado Dr. Aarão de Carvalho (Biopsia No 23.233) revelou estenose segmentar da luz ileal distal, de $7 \mathrm{~cm}$ de extensão, devido a processo esquistossomótico (figura 3 ).

Cerca de 30 dias após, o doente faleceu em choque hipovolêmico e anemia aguda decorrente de enterorragia fulminante.

\section{COMENTÁRIOS}

Pinto e Almeida (16) dividem as manifestaçōes mórbidas da esquistossomose mansônica em dois grandes grupos: formas clínicas e formas cirúrgicas. Nestas, fazem referência onze pacientes de Tavares (23), portadores de retite estenosante associada a parasitose em tela, nos quais em cinco realizaram a amputação retal pela técnica de Miles, com recuperação total.

Meira (12) relata que as formas clínicas com sintomas de obstrução intestinal são excepcionais.

Neves (15) salienta entre as formas intestinais crônicas, a. retite estenosante e a pseudoneoplásica, que por sua importância clínica podem sugerir lesões neoplásicas, notadamente em virtude da sintomatologia de obstrução e de compressōes extrínsecas do intestino.

Raso e Bogliolo (17) distinguem duas formas intestinais, as lesões intestinais ligadas a fase aguda da parasitose e as lesões intestinais da fase crônica. Nesta, classifica em quatro categorias:

a) Formas intestinais hiperplásticas (polipóide, pseudo-neoplásica e reação hiperplástica difusa sub-peritonial);

b) Forma catarral crônica;

c) Fcrma ulcerosa crônica;

d) Forma estenosante.

Fazem referência a sete casos (cinco já descritos por Bicalho (6) da forma pseudo-blastomatosa. 


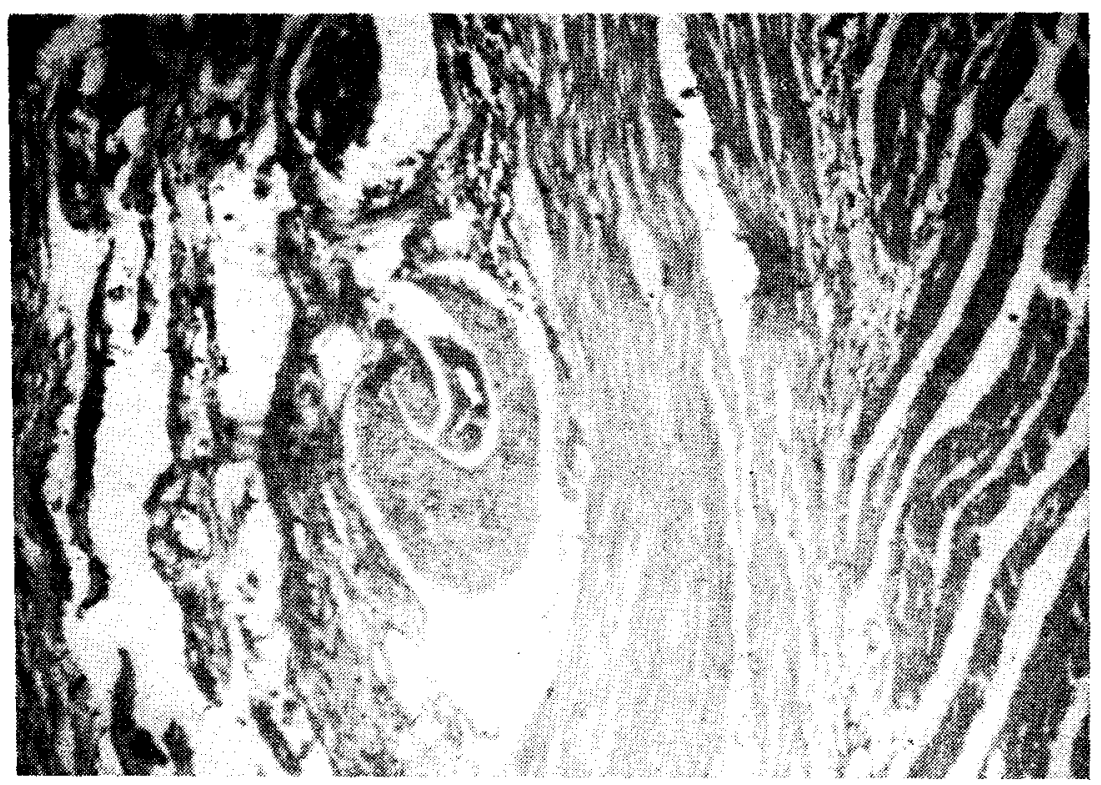

Figura 3 - Paciente S.T.L. - Exame histcpatológico ca peça cirúrgica. Veri.icar a presença de vermes adultos acasalados de Schıstosima manscni (N. 2E E. 400 X)

Tavares (22), relatando os aspectos anatomo-clínicos da esquistossomose, faz menção a casos de colite crônica que $\mathrm{cm}$ vez de diarréia, verifica-se obstipação rebelde, nos quais a luz intestinal se acha reduzida, fibrosada, podendo simular uma neoplasia.

Barreto (4) descreve o caso singular de um paciente portador de esquistossomose aguda e abdome agudo, decorrente de obstrução intestinal alta. Durante a laparotemia encontrou alças jejunais aderentes em grande extensão e serosa espessada e granulosa. O estudo histopatológico revelou ileite esquistossomótica com peritonite.

Valladares (24) classifica as lesões intestinais observadas na esquistossomose mansônica em: a) predominantemente congéstivas; b) predominantemente exsudativas, e c) predominantemente hiperplásticas (incluindo pólipo, a fibrose segmentar estricturante, granuloma pseudo-tumoral, forma tubercular da serosa e a fibrose difusa do intestino). Em sua documentação apresenta dois casos de fibrose estricturante segmentar, um de localização no colo descendente e o outro no reto.

Andrade e Rodrigues (2) descrevem dois enfermos portadores de obstrução intestinal crônica, os quais foram considerados clínica e radiologicamente como possuidores de carcinoma da sigmóide, porém o exame histopatológico das peças operatórias revelou tão somente processo inflamatório produzido pelo Schistosoma mansoni.

Mota (13) apresenta o caso de um doente portador de tumoração localizada no ceco, a qual mostrou-se tratar-se de etiologia esquistossomótica após estudo anátomo- patológico.

Carreño e Diez (8) relataram três doentes com processos de neoformações tumorais de etiologia esquistossomótica (bilharziomas) do grosso intestino.

Celli (9) cita um caso com estenose na porção inferior da sigmóide, encontrando histologicamente abundantes ovos de Schistosoma mansoni nas camadas mucosa $\mathrm{e}$ submucosa.

Mynors (14) descreve um paciente com quadro clínico semelhante a ileite regional (doença de Chron), porém o estudo histológico revelou processo inflamatório crônico granulomatoso de origem esquistossomótico.

Bellard e Carreño (5) em uma revisão de 158.993 histórias clínicas de pacientes cirúrgicos, admitidos em vários Hospitais de Caracas, durante um período de 11 anos, verificaram que cerca de 188 doentes tinham tumores do colo e reto $(0,22 \%)$, desses, 28 casos eram tumores de natureza inflamatória ou granulomatosa e em 110 
tumor era de etiologia esquistossomótica (bilharziomas).

Sobrinho e Kelsch (19) relataram três casos de tumoração intestinal, nos quais o diagnóstico clínico-radiológico foi de lesão neoplásica malígna. Entretanto, o laudo histopatológico revelou a condiçäo esquistossomótica das lesóes.

Armbrust e cols. (3) descreveram 0 caso de um enfermo com quadro de abdome agudo, decorrente de estenose do intestino delgado, o qual foi submetido a laparatomia exploradora com ressecção de $9 \mathrm{~cm}$ de comprimento.

O exame histopatológico da peça operatória mostrou grande espessamento da parede intestinal por inflamação crônica fibrosante de natureza esquistossomótica.

Ferreira (10) relata dois casos cujos aspectos clínicos e radiológicos simulavam uma neoplasia mal'gna. o exame histopatológico mo trou a natureza esquistossomótica das lesões.

Stock e Li (21), ao estudarem as lesões granulomatosas do intestino que simulam tumores malígnos, descreveram se $s$ casos de etiologia benígna, um dos quais de origem esquistossomótica.

Bicalho (6) apresenta cinco casos da forma pseudo-tumoral da esquiztojromose mansoni e discute a gênese dessas formacôes conjuntivo-hiperplásticas, estabelecendo uma classificação levando $\mathrm{em}$ cousideração a sede e a sua localização na parede intestinal. Dessa maneira, subdividem essas formações conjuntivas hiperplásticas (pseudo-tumorais) em: intestinais e extra-intestinais. As relacionadas com o intestino podem ser sediadas: exclusiva ou pre- dominante na submucosa, exteriorizando para a luz intestinal (da forma intestinal interna) e na subserosa, crescendo para a superficie externa do intestino (forma intestinal externa).

Stephens (20) relata o caso de granuloma bilharziano, o qual se apresentava clinicamente como uma massa tumoral palpável no quadrante inferior esquerdo do abdome.

Ribeiro Jorge e cols. (18) apresentam um caso de suboclusão intestinal (ileo distal), com quadro de abdome agudo determinado por enterite granulomatosa esquistossomótica (comprovada pelo estudo aná tomo-patológico da peca cirúrgica).

Bicalho (7) descreve um caso da forma pseudo-neoplástica da esquistosimose mansônica, localizada no espaço retroperitonial, cuja evolução rápida sugeriu a hipótese de neoplasia maligna a despeito da boa aparência geral do paciente.

Álvaro e cols. (1) aprisentam um paciente com tumoração abdominal, localizada ao nivel do hipogastro e fossa iliaca esquerda, medindo cerca de $8 \times 2 \mathrm{~cm}$ e indolor. O exame histopatológico da peça cirúrgica mostrou formação pseudo-tumoral de natureza esouistossomótica da região sigmoidiana, retosigmoidite e linfadenite crônica esquistossomótica.

Katz (11) relata quatro casos da forma Gumoral da esquisto somose mansônica, todos com diagnóstico histopatológico.

Zeitune e cols. (25) apresentam um caso da forma pseudo-neoplásica da esquistossomose mansônica, com localização retal, cujo diagnóstico clínico sugeriu o de neoplasia maligna.

\section{$S U M M A R Y$}

The Author reports a case of stenosis of the rmall bowel (distal ileum) wich surgical specimen sinnulated the aspects produced of carcinoma, but the histological st!dy (microscopic findings) showed inflammation due to Schistosomiasis mansoni. 


\section{REFTRENCIAS BIBLIOGRÁFICAS}

1 - Allvaro, P. et alii - Manifestações raras da esquistossomose mansônica. An. Fac. Med. Univ. Fed. Pe., 28/29: 15-24, 1968/1969.

2 - ANDRADE, Z.A. \& RODRIGUES, G. Manifestações pseudo-neoplásticas da esquistossomose intestinal. Arq. Bras. Med., 44: 437-444, 1954.

3 - AMBRUST, A.F. et alii - Granuloma esquistossomótico do intestino delgado. An. Paul. Med. Cir., 82: 195-196, 1961.

4 - BARRETO, A.M. - Esquistossomose aguda - abdome agudo - obstrução intestinal por granuloma esquistossomótico. Brasil-Médico, 67: 420-422, 1953.

5 - BELLARD, E.P. \& CARREÑO, P. Granulomatous tumors and neoplasms of the colon and anorectal zone in Venezuela. Surgery, 44: 627$-630,1958$.

6 - BICALHo, S.A. - Sobre as neoforma ções conjuntivo-hiperplásticas pseudo-tumorais na esquistossomose mansoni. G.E.N. (Caracas), 19: 257-271, 1965.

7 - BICALHO, S.A. - Pseudo neoplastic form of Schistosomiasis mansoni Gastroenterology, 54: 105-109, 1968.

8 - CARRENTO, M.P. \& DIEZ, A. - Las neoformaciones bilharzianas del intestino grueso (Los bilharziomas). G.E.N. (Caracas), 10 (No Extraordinário): 603-614, 1955.

9 - CELLI, B.B. - Lesiones anatomopatologicas de la bilharziosis en cole y recto. G.E.N. (Caracas), 10 (No Extraordinário): 519-524. 1955.

10 - FERREIRA, L.C. - Colopatias esquistossomóticas pseudo-carcinomatosas. Arq. Oncol., 4: 165-171, 1961.
11 - KATZ, N. - Resposta ao questionário: Como se apresentam seus casos de forma tumoral da esquistossomosea An. LL Simpósio solure Esquistossomose. Prata, A. e Aboim, E. (Editores), Salvador, 1970, pp. 186-190 .

12 - MEIRA, J.A. - Esquistossomose mansônica. In Veronesi, R. (Editor) - Doenças Infecciosas e Parasitárias, E d i t o r a Guanabara-Koogan, Guanabara, Rio de Janeiro, 1962, pp. 645-702 .

13 - MOTA, J.N. - Tumor do ceco por esquistossomose. Rev. Assoc. Med. M. G., 5: 69-70, 1954.

14 - MYNORS, J.M. - Intestinal Schistosomiasis resembling regional ileitis. Trans. Roy. Soc. Trop. Med. Hyg., 51: 45-47, 1957.

15 - NEVES, J. - Quadro clínico da esquistossomose mansoni. In Cunha, A. S. - Esquistossomose mansoni. Editora da Universidade de São Paulo, São Paulo, 1970, pp. 131-191.

16 - PINTO, C. \& ALMEIDA, A.F. - Formas clínicas da esquistossomose mansoni no Brasil. Rev. Bras. Med., 2: 636-652, 1945.

17 - RASO, P. \& BOGLIOLO, L. - Patologia da esquistossomose mansoni. In Cunha, A. S. - Esquistossomose mansoni. Editora da Universidade de São Paulo, São Paulo, 1970, pp. 77-130 .

18 - RIBEIRO JORGE, P.A. et alii Obstrução intestinal por granuloma esquistossomótico. Apresentação de um caso com estudo clínico-patológico. Rev. Assoc. Med. Bras., 13: 147-148, 1967.

19 - SOBRINHO, J. \& KELSCH, F.O. Aspectos tumorais da esquistossomo- 
se do colon. Rev. Bras. Radiol., 2: 23 - TAVARES, L. -Comentários sobre a 1-7, 1959.

20 - STEPHENS, R.R. - Bilharziasis as a differential diagnosis for abdominal tumors. Cent. Afr. J. Med., 12: 28-29, 1966.

21 - STOCK, F.E. \& LI, F.W.P. Granuloma of the large/bowel simulating malignant disease. Brit. J. Surg., 51: 898-901, 1964.

22 - TAVARES, A.S. - Aspectos anátomo-clínicos da Schistosomose. Brasil-Médico, 49: 801-812, e 823-835, 1935. patogenia da esquistossomose mansônica, 1943. (Citado por Pinto e Almeida, 16) .

24 - VALLADARES, C.P. - Determinações intestinais da doença de Manson-Pirajá da Silva. Tese, Faculdade de Medicina Universidade da Bahia, 1953.

25 - ZEITUNE, J.M.R. et alii - Forma pseudoneoplástica da esquistossomose mansoni. Apresentaçāo de um caso autóctone do Norte do Paraná. $\mathrm{X}$ Congresso da Sociedade Brasileira de Medicina Tropical, Curitiba, 1974. 